\title{
Inhibition of NF- $\kappa$ B Reduces Renal Inflammation and Expression of PEPCK in Type 2 Diabetic Mice
}

\author{
Qianling Liu, ${ }^{1}$ Liangyan Zhang, ${ }^{1}$ Wei Zhang, ${ }^{1}$ Qiufa Hao, ${ }^{1}$ Wei Qiu, ${ }^{1}$ Yubing Wen, \\ Haiyun Wang, ${ }^{1}$ and Xuemei $\mathrm{Li}^{1,2}$
}

\begin{abstract}
Renal gluconeogenesis is markedly promoted in patients with type 2 diabetes mellitus (T2DM); however, the underlying mechanism remains largely unknown. Renal gluconeogenesis is found to be negatively regulated by insulin. T2DM is characterized by chronic and subacute inflammation; however, inflammation has been well recognized to induce insulin resistance. Therefore, this study aimed to investigate whether the enhanced renal gluconeogenesis in T2DM was partially due to the renal inflammation-mediated insulin resistance. If so, whether inflammation inhibitor could partially reverse such change. Diabetic $\mathrm{db} / \mathrm{db}$ mice and $\mathrm{db} / \mathrm{m}$ mice were used in our study. Typically, diabetic $\mathrm{db} / \mathrm{db}$ mice were intraperitoneally treated with $1 \mathrm{mg} / \mathrm{kg} \mathrm{NF}-\mathrm{kB}$ inhibitor parthenolide (PTN) or saline as control every other day. Twelve weeks after treatment, animal samples were collected for measurements. Our results suggested that the expression levels of the inflammatory factors and the gluconeogenic rate-limiting enzyme phosphoenolpyruvate carboxykinase (PEPCK) were upregulated in renal cortex of both $\mathrm{db} / \mathrm{db}$ mice and T2DM patients. Moreover, reduced insulin signaling, as well as up-regulated expression of downstream genes FOXO1 and PGC-1a, could be detected in renal cortex of $\mathrm{db} / \mathrm{db}$ mice compared with that of $\mathrm{db} / \mathrm{m}$ mice. Consistent with our hypothesis, PTN treatment could alleviate renal inflammation and insulin resistance in $\mathrm{db} / \mathrm{db}$ mice. Moreover, it could also down-regulate the renal expression of PEPCK, indicating that inflammation could be one of the triggers of insulin resistance and the enhanced renal gluconeogenesis in $\mathrm{db} / \mathrm{db}$ mice. This study can shed light on the role of inflammation in the enhanced renal gluconeogenesis in T2DM, which may yield a novel target for hyperglycemia.
\end{abstract}

KEY WORDS: inflammation; insulin resistance; renal gluconeogenesis; type 2 diabetes mellitus.

\section{INTRODUCTION}

The worldwide prevalence of type 2 diabetes mellitus (T2DM) is increased dramatically, which has thereby

\footnotetext{
${ }^{1}$ Department of Nephrology, Peking Union Medical College Hospital, Chinese Academy of Medicine Sciences and Peking Union Medical College, Beijing, 100730, China

${ }^{2}$ To whom correspondence should be addressed at Department of Nephrology, Peking Union Medical College Hospital, Chinese Academy of Medicine Sciences and Peking Union Medical College, Beijing, 100730, China. E-mail: lixmpumch@126.com
}

caused serious health consequences. Excessive glucose release into the blood is viewed as a main cause responsible for hyperglycemia in T2DM [1], and the enhanced renal gluconeogenesis has gradually attracted increasing attention. Historically, renal gluconeogenesis is suggested to notably contribute to the systemic glucose release merely after prolonged starvation or acidosis [2]. Nonetheless, its role is shown to be changed in recent decades [3-5]. Physiologically, gluconeogenesis in both the kidneys and liver will offer approximately equal amounts of glucose $(20-25 \%)$ to the body in fasting state. Besides, the increase 
in fasting frequency will lead to the increased systemic glucose production ratio as a result of renal gluconeogenesis [6]. More surprisingly, renal gluconeogenesis is almost doubled in postprandial state, which accounts for about $60 \%$ of systemic glucose release [5]. However, renal gluconeogenesis is altered in T2DM patients, specifically, it is increased in T2DM in the fasting state [7]. Previously, the liver is deemed as the main organ responsible for the enhanced glucose release into the blood in T2DM patients. In fact, the absolute increase in glucose release from the kidney is comparable to that from the liver (kidney $2.21 \mathrm{vs}$ liver $2.60 \mu \mathrm{mol} /(\mathrm{kg} \mathrm{min}) ; P=0.26)$ [7]. Additionally, the relative increase in gluconeogenesis in the kidney is much higher than that in the liver (300 vs 30\%) [7]. Postprandial renal gluconeogenesis is also substantially enhanced in T2DM patients compared with that in normal subjects [8]. These studies have emphasized the magnitude and importance of renal gluconeogenesis. However, the exact mechanism of the enhanced renal gluconeogenesis in T2DM remains unclear yet.

Renal gluconeogenesis is found to be negatively modulated by insulin both in animals and humans [911]. Studies on human hyperinsulinemic clamp demonstrate that insulin can reduce about $61 \%$ of renal gluconeogenesis [12]. Therefore, insulin resistance can theoretically lead to the enhanced renal gluconeogenesis. As has been well established, inflammation can directly induce insulin resistance. A century ago, doctors accidentally found that high doses of sodium salicylates could reduce the blood glucose levels in T2DM patients, which had provided a crucial clew for the association between inflammation and the pathogenesis of T2DM. However, the anti-inflammatory and antihyperglycemic effects of sodium salicylates have not been linked to insulin resistance until now. In company with the finding of tissue macrophage infiltration, these novel discoveries contribute to correcting the relationship between inflammation and insulin resistance [13]. Inflammatory stimulation can induce insulin resistance by stimulating the activation of the JNK and NF-KB pathways, while T2DM is characterized by the chronic and subacute inflammation as well as insulin resistance [14, 15]. Thus, it was speculated in the current study that the enhanced renal gluconeogenesis in T2DM was partially resulted from the renal inflammation-mediated insulin resistance. Moreover, we also examined whether treatment of inflammation inhibitor could partially reverse such change. To verify our hypothesis, the $\mathrm{db} / \mathrm{db}$ mice, which were considered to be the excellent T2DM models, were used in this study.

\section{MATERIALS AND METHODS}

\section{Chemicals and Antibodies}

Antibodies against F4/80, NF- $\mathrm{kB}$ p65, phosphoenolpyruvate carboxykinase (PEPCK), glucose 6 phosphatase (G6Pase), 6-bis phosphatase (FBPase), forkhead transcription factor1 (FOXO1), peroxisome proliferator-activated receptor gamma coactivator 1-alpha (PGC-1a), Ser473phosphorylated AKT, AKT, and $\beta$-actin were purchased from Abcam. Antibody against $8-\mathrm{OHdG}$ was obtained from Santa Cruz Biotechnology. Other materials were all purchased commercially and were of high analytical quality.

\section{Patients and Tissue Samples}

Normal human kidney tissue samples were acquired from the nondiabetic patients undergoing radical nephrectomy for renal carcinoma at the Urology Department of Peking Union Medical College Hospital with patient consent in accordance with the hospital policy. These tissue samples were identified as normal by an expert nephropathologist. Renal biopsy specimens from T2DM patients were acquired from the Pathology Department of Peking Union Medical College Hospital with patient consent in line with the hospital policy.

\section{Animal Model}

The 8-week-old male db/db T2DM mice (C57BLKS/ $\mathrm{J}-\mathrm{LepR}^{\mathrm{db}} / \mathrm{LepR}^{\mathrm{db}}$ ) and the nondiabetic littermate $\mathrm{db} / \mathrm{m}$ mice $\left(\mathrm{C} 57 \mathrm{BLKS} / \mathrm{J}-\mathrm{LepR} \mathrm{Rb}^{\mathrm{db}+}\right.$ ) were purchased for study in vivo. Diabetic $\mathrm{db} / \mathrm{db}$ mice were given intraperitoneal injection of $1 \mathrm{mg} / \mathrm{kg}$ parthenolide (PTN), the NF-KB inhibitor, or saline as control every other day. After treatment of $0,4,8$, and 12 weeks, the kidney, blood, and urea specimens were collected for measurements following all national and institutional guidelines for the care and use of animals.

\section{Blood Examinations}

Blood samples were collected every 4 weeks. Blood glucose levels were detected using the glucose test kit. In addition, serum insulin, cholesterol, and triglyceride were measured through the commercial kit (PUMCH Laboratory). 


\section{Real-time Quantitative PCR}

Real-time quantitative PCR (RT-qPCR) was performed on the 7500 Fast Real-Time qPCR System using GoTaq® qPCR Master Mix (Promega, Madison, USA). Primer sequences used in this study are shown in Table 1, while $\beta$-actin was used as the reference gene.

\section{Western Blotting}

RIPA lysis buffer (Millipore, Bedford, MA) was used for tissue lysis. Subsequently, equivalent amounts of protein samples were separated through SDS/PAGE (10-12\% gel) and transferred onto the polyvinylidene fluoride (PVDF) membranes. After blocking with 5\% bovine serum albumin (BSA), membranes were incubated with primary antibodies at $4{ }^{\circ} \mathrm{C}$ overnight, followed by incubation with HRP-conjugated secondary antibodies for $1 \mathrm{~h}$. Reactions were visualized with electrochemiluminescence (ECL) and chemiluminescence using the ChemiDoc XRSp system (Bio-Rad). Western blotting was quantitatively analyzed using the Image $\mathrm{J}$ software and normalized by the actin level.

\section{Immunohistochemistry Assays}

The paraffin-embedded human renal tissues were deparaffinized and rehydrated in graded ethanol. Afterwards, the tissue sections were placed in a microwave for antigen retrieval and blocked with $10 \%$ goat serum. Sections were then incubated with specific primary antibody at $4{ }^{\circ} \mathrm{C}$ overnight, followed by incubation with secondary antibody for $30 \mathrm{~min}$ at room temperature [16]. Positive staining was viewed under the light microscope using DAB substrate. Additionally, immunohistochemical quantitative analysis was performed using ImagePro Plus 6.0. Data were shown as the fold change of average IOD per area.

\section{Statistical Analysis}

All data were expressed as mean $\pm \mathrm{SD}$, and a difference of $P<0.05$ was considered as statistically significant. Comparison between two groups was analyzed using the two-tailed $t$ test, while differences among multiple groups were assessed by the one-way analysis of variance (ANOVA).

\section{RESULTS}

\section{Expression of NF- $\kappa \mathrm{B}$ and Gluconeogenic Enzymes in T2DM Human Kidney}

Up-regulated expression of NF- $\mathrm{KB}$ and the gluconeogenic rate-limiting enzyme PEPCK could be detected in the renal cortex of T2DM patients compared with the nondiabetic controls (Fig. 1), but the expression of fructose 1, 6-bis phosphatase, and glucose 6 phosphatase was not up-regulated. In addition, biopsies from T2DM patients revealed the redistribution of FBPase and PEPCK to the lumen membrane of proximal tubule (PT).

\section{Metabolic Parameters in Experimental Animals}

Diabetic $\mathrm{db} / \mathrm{db}$ mice were used in the current study to test the role of inflammation in the enhanced renal gluconeogenesis of T2DM, and the NF- $\mathrm{KB}$ inhibitor parthenolide was employed to alleviate inflammation. The structure of PTN is shown in Fig. 2a. The 8-week-old db/db T2DM mice had already developed obesity, hyperglycemia, insulin resistance (HOMA-IR), and hyperlipidemia in comparison with the control $\mathrm{db} / \mathrm{m}$ mice, as shown in Fig. $2 \mathrm{~b}-\mathrm{g}$. PTN could slightly reduce the blood glucose and HOMAIR levels in $\mathrm{db} / \mathrm{db}$ mice at the age of 16 and 20 weeks. However, there were no statistically significant differences among these three groups. PTN made no difference to the

Table 1. Real-time PCR Primers Used in the Study

\begin{tabular}{|c|c|c|}
\hline Gene name (mouse) & Forward $\left(5^{\prime}-3^{\prime}\right)$ & Reverse $\left(5^{\prime}-3^{\prime}\right)$ \\
\hline ICAM-1 & TGGCCTGGGGGATGCACACT & GGCTGTAGGTGGGTCCGGG \\
\hline MIP-1 $\alpha$ & СTTCTCTGTACCATGACACTCTGC & ATTCAGTTCCAGGTCAGTGATGTAT \\
\hline $\mathrm{TNF}-\alpha$ & AGCCCCCAGTCTGTATCCTT & CTCCCTTTGCAGAACTCAGG \\
\hline PEPCK & TGCGGATCATGACTCGGATG & AGGCCCAGTTGTTGACCAAA \\
\hline G6pase & TTTCCCCACCAGGTCGTGGCT & CCCATTCTGGCCGCTCACAC \\
\hline FBPase & ATGGTATCGCTGGCTCAACC & ACAGGTAGCGTAGGACGACT \\
\hline FOXO1 & AAGCTCCCAAGTGACTTGGATG & CTGCTCACTAACCCTCAGCCTGA \\
\hline PGC- $1 \alpha$ & AAGTGTGGAACTCTCTGGAACTG & GGGTTATCTTGGTTGGCTTTATG \\
\hline$\beta$-actin & TGTTACCAACTGGGACGACA & GGGGTGTTGAAGGTCTCAAA \\
\hline
\end{tabular}


a
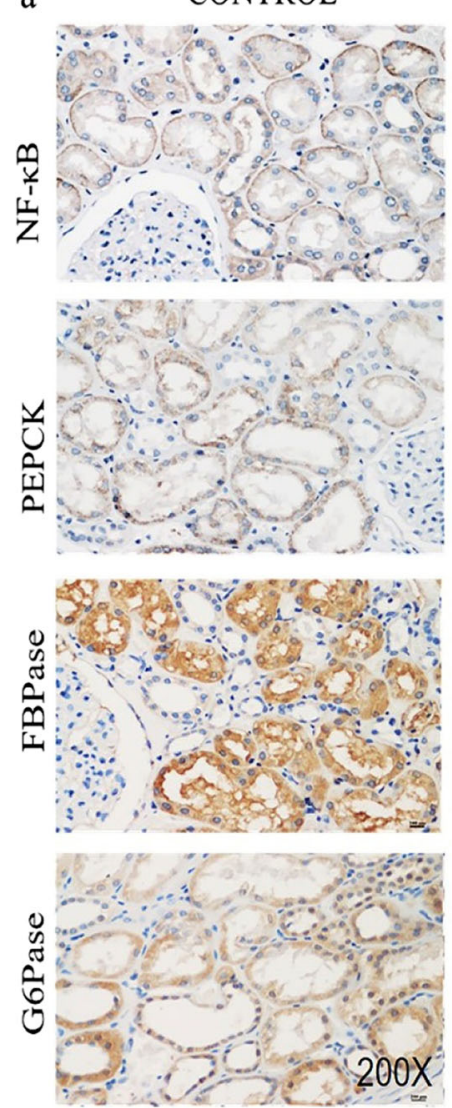
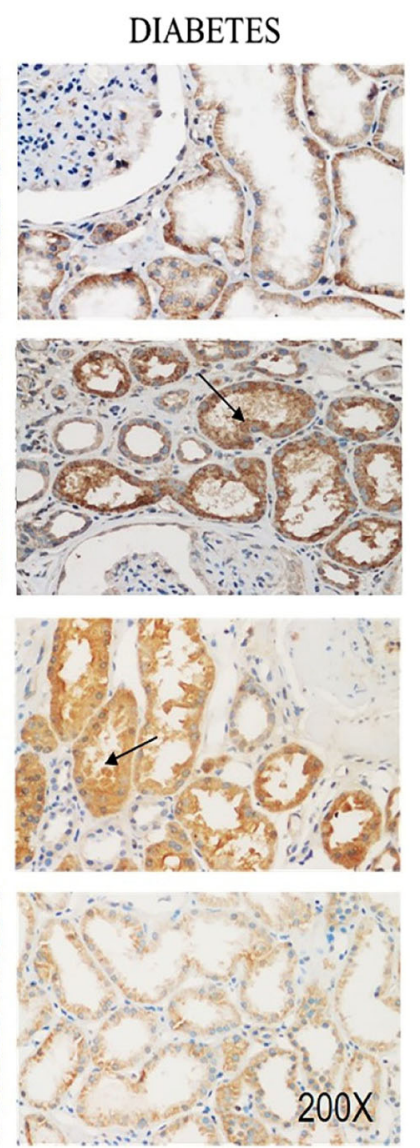
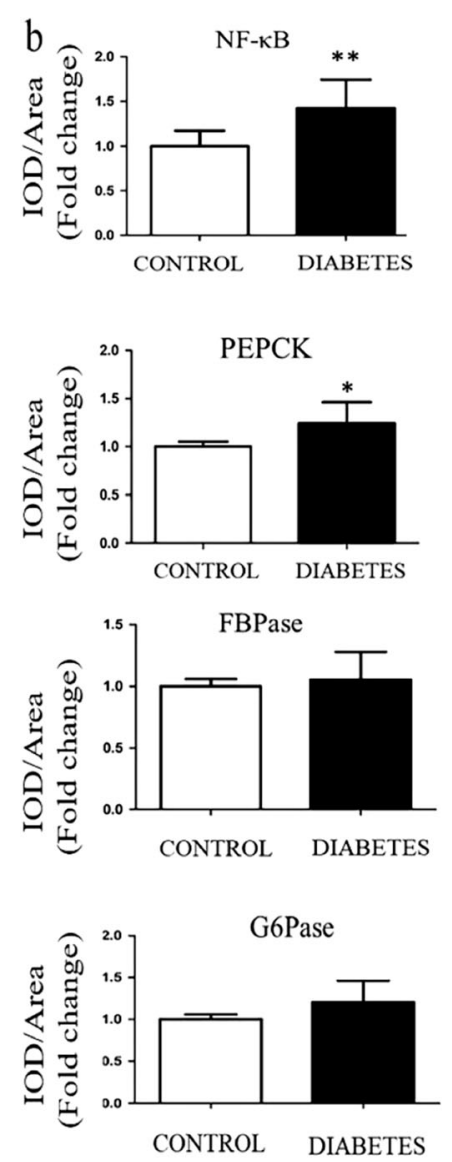

Fig. 1. Expression of NF- $\mathrm{KB}$ and gluconeogenic enzymes in human kidney. a Immunohistochemistry of NF- $\mathrm{KB}$ and gluconeogenic enzymes from normal control and patients with T2DM. Black arrows - apical distribution. Magnification: $\times 200$. $\mathbf{b}$ Relative fold change of IOD/area (control, $n=7$; diabetes, $n=$ 20). $* P<0.05$ and $* * P<0.01$.

body weights of mice (Fig. 2b). Besides, no statistical differences were observed in serum uric acid among all three groups (Fig. 2h).

\section{Renal Expression of Inflammatory Mediators in Diabetic Mice}

In $\mathrm{db} / \mathrm{db}$ mice, the renal expression levels of NF$\mathrm{\kappa B}$ and its downstream inflammatory mediators, including intercellular adhesion molecule-1 (ICAM-1), tumor necrosis factor alpha (TNF-a), and macrophage inflammatory protein 1 alpha (MIP-1 $\alpha$ ), were remarkably enhanced relative to those in $\mathrm{db} / \mathrm{m}$ control mice (Fig. 3a-c). Additionally, PTN could down-regulate the renal expression of these inflammatory mediators in $\mathrm{db} / \mathrm{db}$ mice (Fig. 3a-c). Moreover, $\mathrm{db} / \mathrm{db}$ mice exhibited more severe renal macrophage infiltration and oxidative stress, as identified by the stronger tissue staining of F4/80 and 8-hydroxydeoxyguanosine (8OHdG) than that in $\mathrm{db} / \mathrm{m}$ mice (Fig. 3d). Besides, PTN could alleviate renal oxidative stress and macrophage infiltration in $\mathrm{db} / \mathrm{db}$ mice (Fig. 3d).

\section{Renal Expression of Gluconeogenic Enzymes in Diabetic Mice}

Consistent with the results in human kidney, $\mathrm{db} / \mathrm{db}$ mice also displayed the enhanced renal expression of PEPCK but not FBPase and G6Pase, relative to that in the $\mathrm{db} / \mathrm{m}$ mice (Fig. 4). Moreover, the redistribution of PEPCK and FBPase to lumen of PT could also be observed in the kidneys of diabetic mice (Fig. 4b). In line with our hypothesis, PTN could markedly down-regulate the renal mRNA and protein expression of PEPCK in $\mathrm{db} /$ 
a

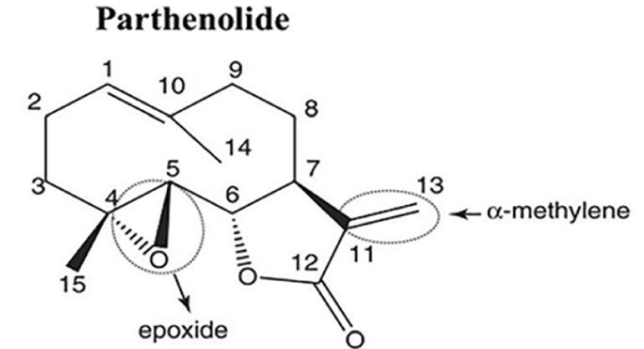

$\mathrm{C}$

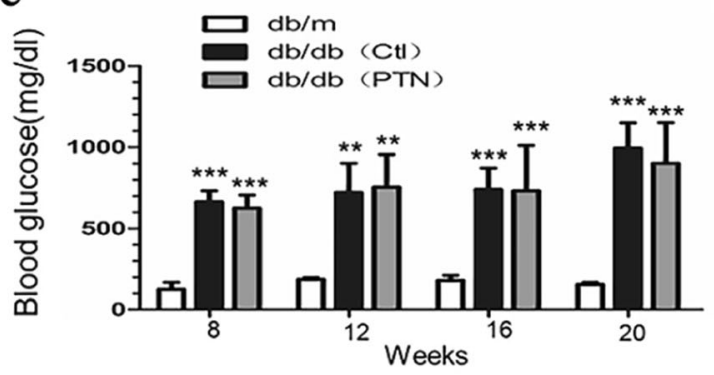

e

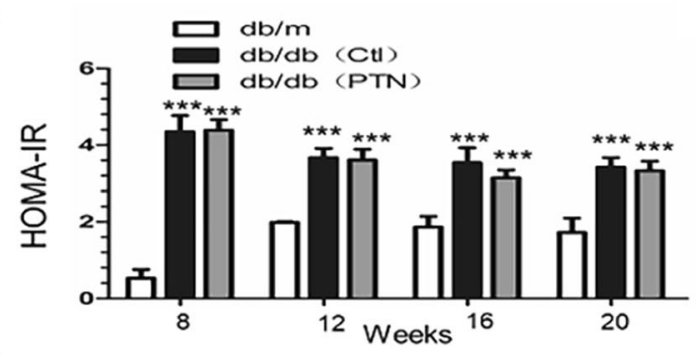

$\mathrm{g}$

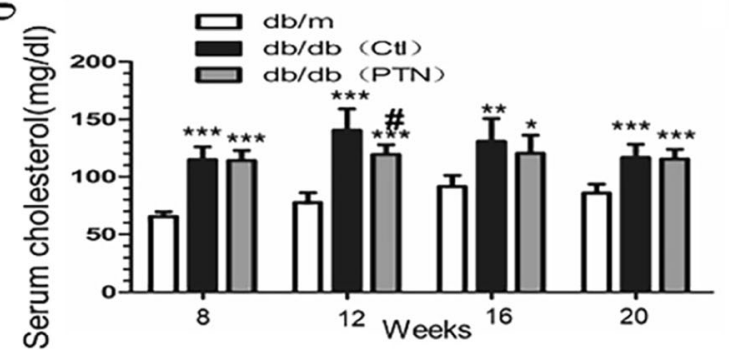

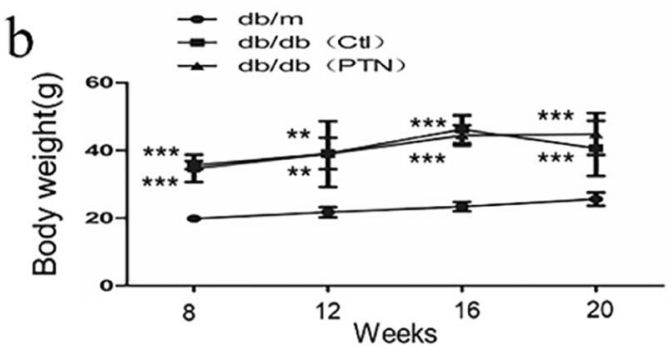

d
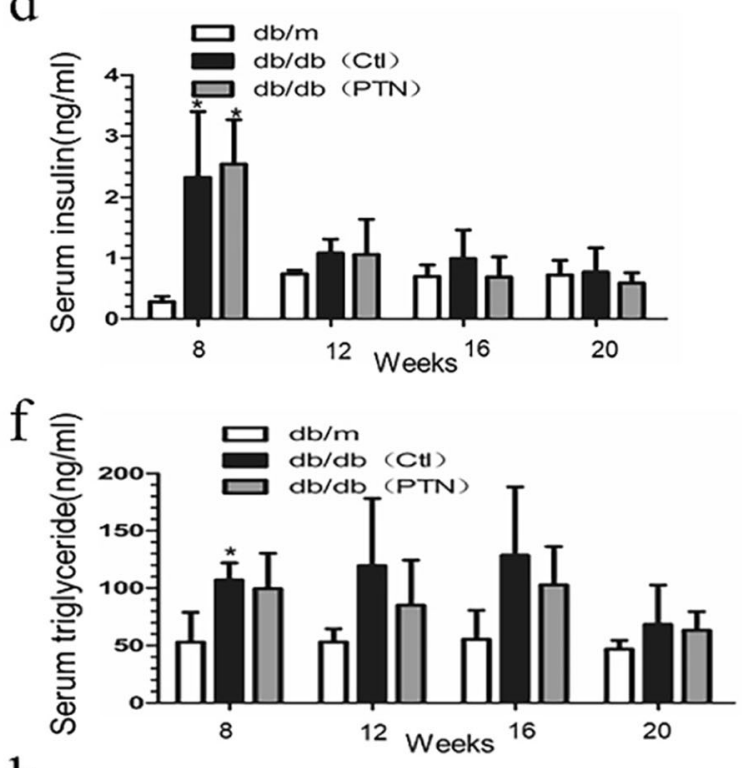

$\mathrm{h}$

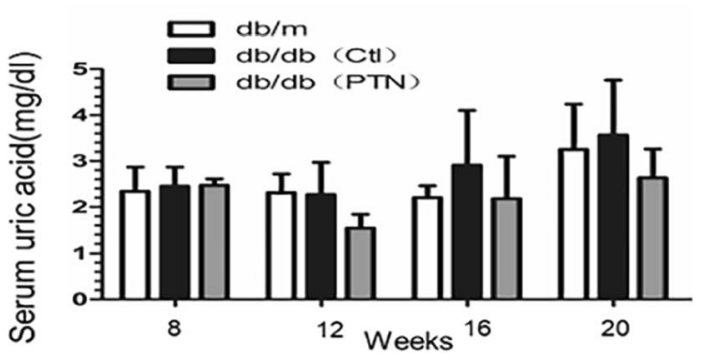

Fig. 2. Metabolic parameters in experimental animals. a Structure of parthenolide (PTN). Changes in b body weight, $\mathbf{c}$ blood glucose, $\mathbf{d}$ serum insulin, $\mathbf{e}$ HOMA-IR, f serum triglyceride, $\mathbf{g}$ serum cholesterol, and $\mathbf{h}$ serum uric acid in $\mathrm{db} / \mathrm{m}$ and $\mathrm{db} / \mathrm{db}$ mice treated without or with PTN for 2 months starting at age of 8 weeks. $* P<0.05 ; * * P<0.01$; and $* * * P<0.001$ vs $\mathrm{db} / \mathrm{m}$ mice; $\# P<0.05$ vs $\mathrm{db} / \mathrm{db}(\mathrm{Ctl})$ mice. $n=6-8$ for each group.

$\mathrm{db}$ mice, indicating that inflammation could be one of the triggers for the enhanced expression of renal gluconeogenic gene (Fig. 4a, c).

\section{Renal Expression of the Insulin Signaling Pathway in Diabetic Mice}

To further examine the relevant insulin signaling cascade in mice kidneys, the insulin-induced activation of AKT was detected. Meanwhile, our attention was centered on FOXO1 and PGC-1a, the two key genes associated with insulin resistance and the dysregulated glucose metabolism in the liver. As shown in Fig. 5, $\mathrm{db} / \mathrm{db}$ mice exhibited increased expression of $\mathrm{p}$-IRS1 (s307) and reduced renal insulin signaling, as demonstrated by the down-regulated phosphorylation of AKT compared with that in $\mathrm{db} / \mathrm{m}$ mice, which could be partially reversed by PTN (Fig. 5b). In addition, db/ 


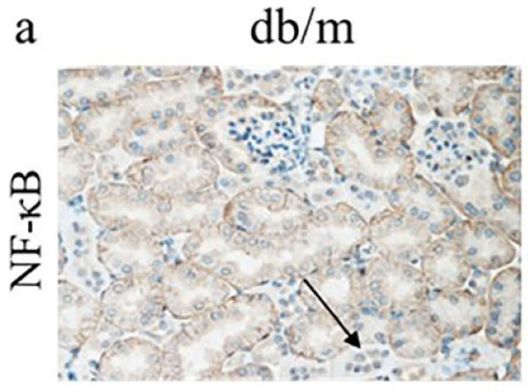

$\mathrm{b}$

$$
\mathrm{NF}-\kappa \mathrm{B}
$$

$\beta$-actin

$\mathrm{c}$
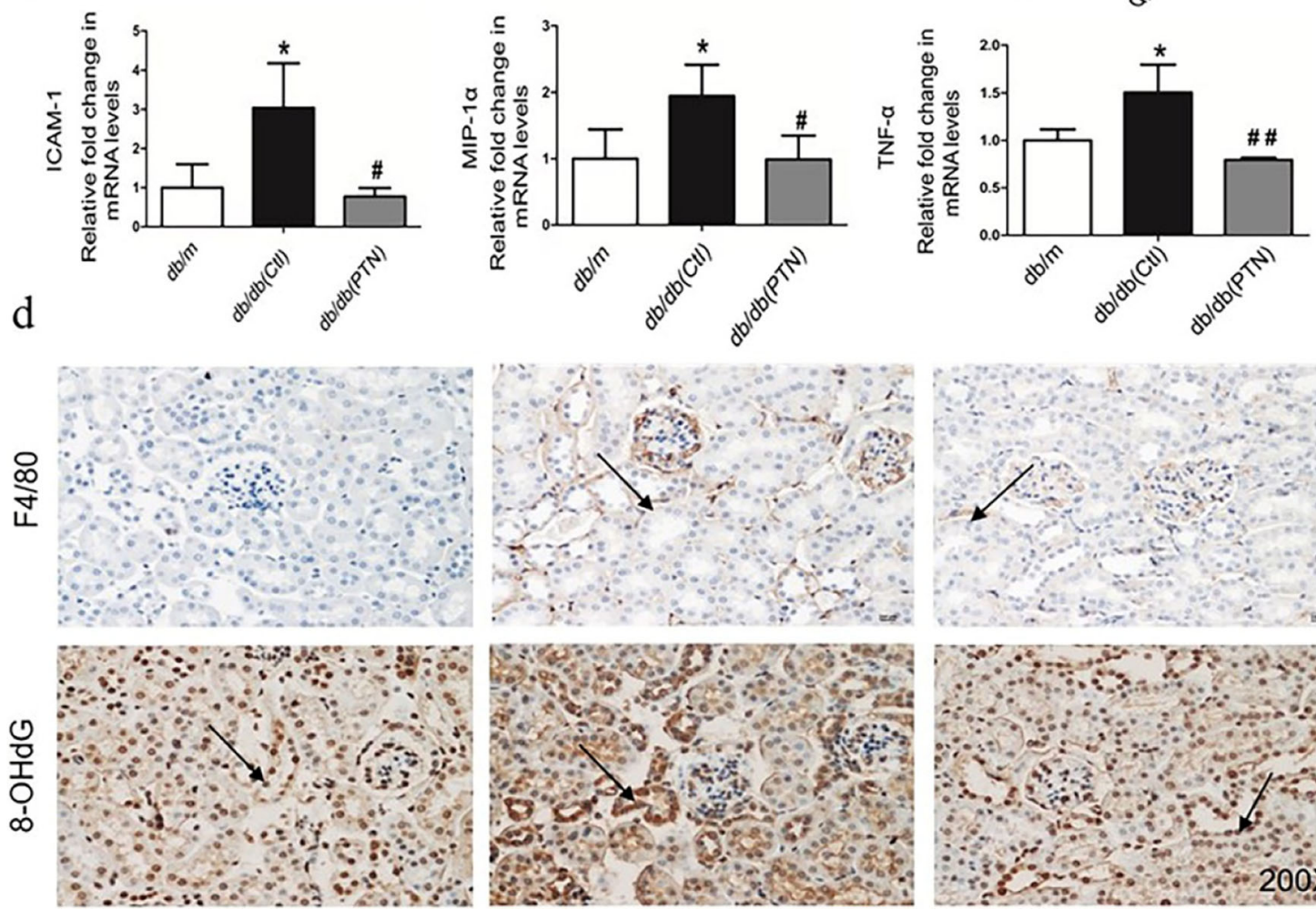

Fig. 3. Expression of inflammatory mediators in diabetic mice kidney. a and $\mathbf{b}$ Immunohistochemistry and western blots of NF- $\mathrm{KB}$ from mice kidney. Black arrows - nuclear translocation of NF-KB. c Real-time PCR analysis of ICAM-1, MIP-1a, and TNF-a in mice kidney. d Immunohistochemical staining of F4/ $80^{+}$cells and 8-OHdG on kidney tissue section. $* P<0.05$ and $* * P<0.01 v s \mathrm{db} / \mathrm{m}$ mice; $\# P<0.05 v s \mathrm{db} / \mathrm{db}(\mathrm{Ctl})$ mice. $n=6-8$ for each group.

$\mathrm{db}$ mice showed higher renal mRNA and protein levels of FOXO- 1 and PGC- 1 a relative to those in $\mathrm{db} / \mathrm{m}$ mice (Fig. 5a). However, PTN did not dramatically downregulate the renal expression of FOXO1 and PGC-1a in $\mathrm{db} / \mathrm{db}$ mice.

\section{DISCUSSION}

Recent studies reveal that renal gluconeogenesis is substantially activated in T2DM patients $[7,8]$, but the precise mechanism remains poorly understood so far. 


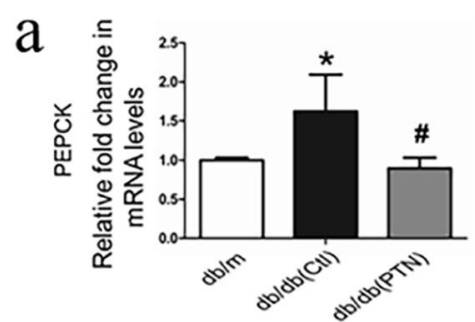

b

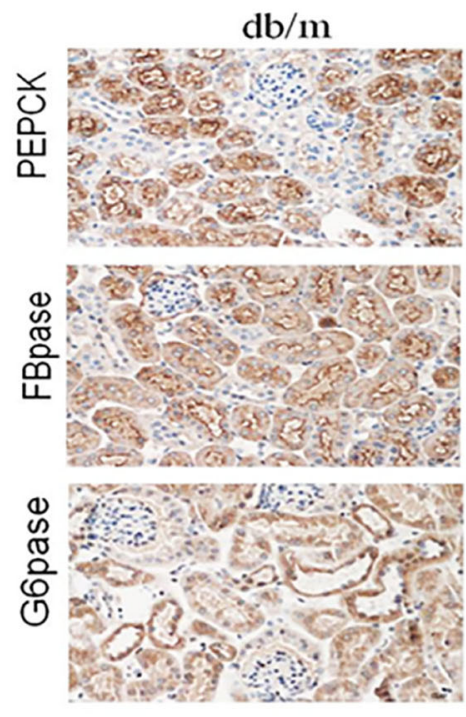

$\mathrm{c}$

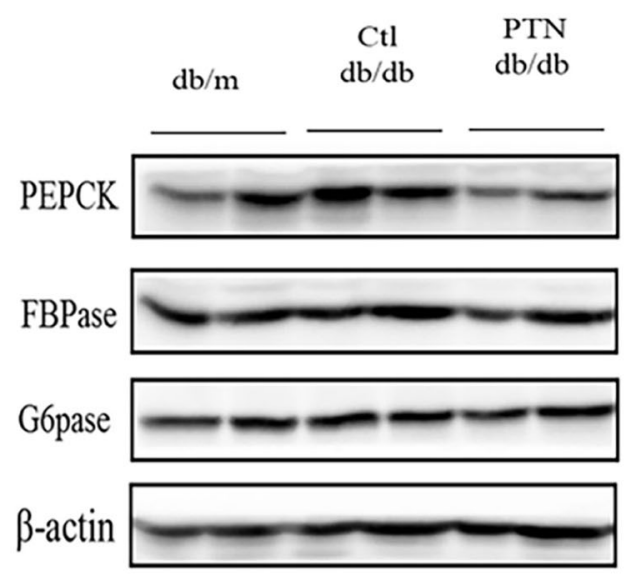

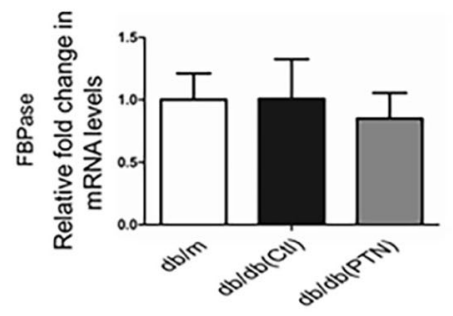

$\mathrm{db} / \mathrm{db}(\mathrm{CTL})$
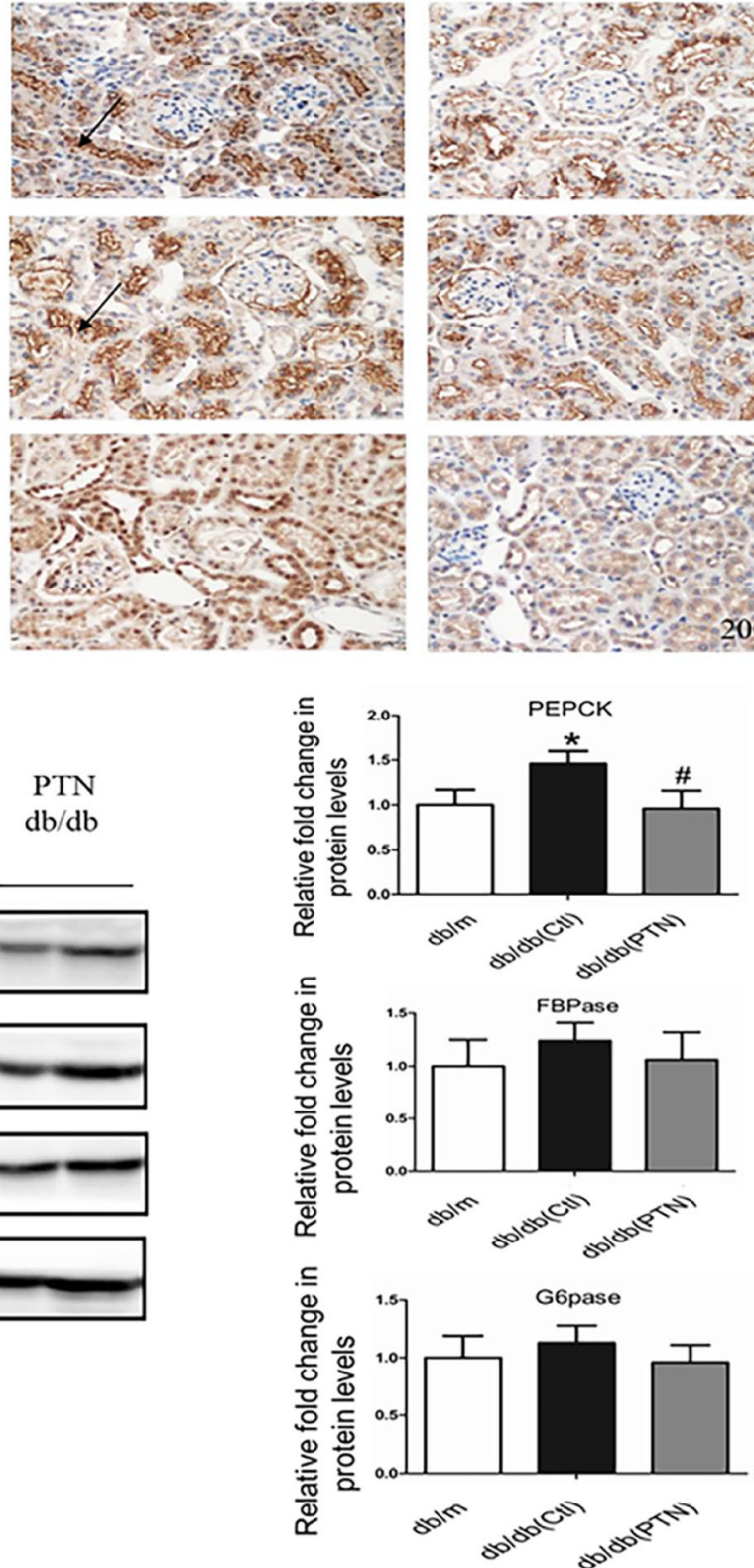

Fig. 4. Expression of gluconeogenic enzymes in mice kidney. a Real-time PCR, $\mathbf{b}$ immunohistochemistry, and $\mathbf{c}$ western blots of gluconeogenic enzymes in renal cortex of $\mathrm{db} / \mathrm{m}, \mathrm{db} / \mathrm{db}(\mathrm{Ctl})$, and db/db (PTN) mice. Black arrows - apical distribution. $* P<0.05$ compared with $\mathrm{db} / \mathrm{m}$; $\# P<0.05 \mathrm{compared}$ with $\mathrm{db} / \mathrm{db}$ (Ctl). $n=6-8$ for each group. 
a

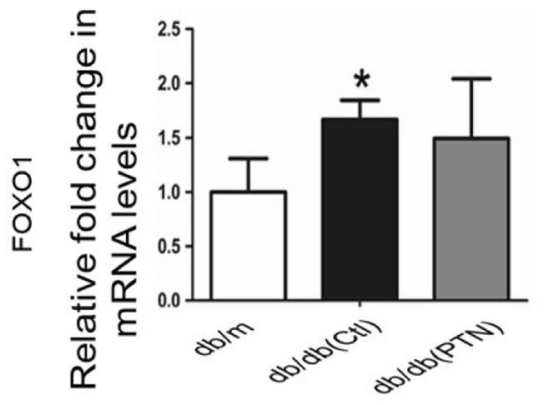

$\mathrm{b}$

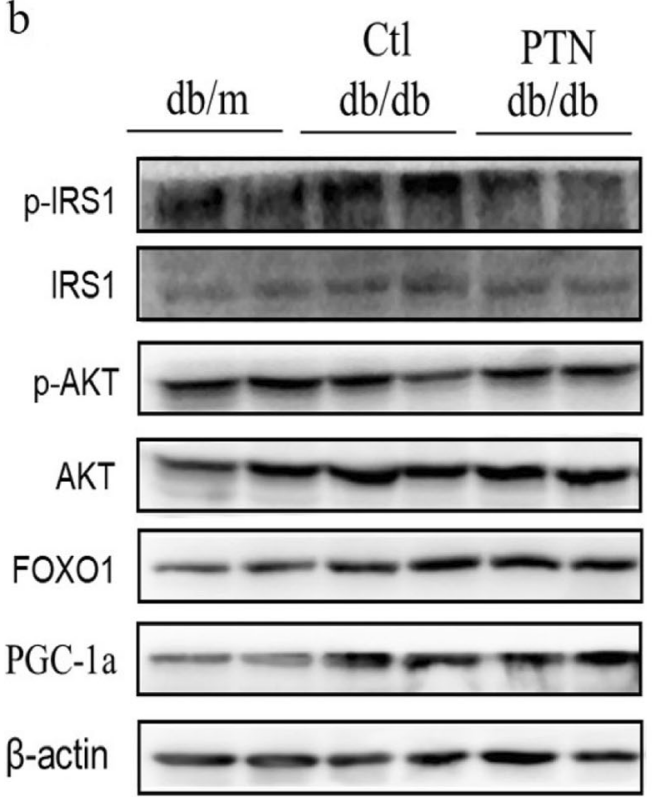

$\beta$-actin
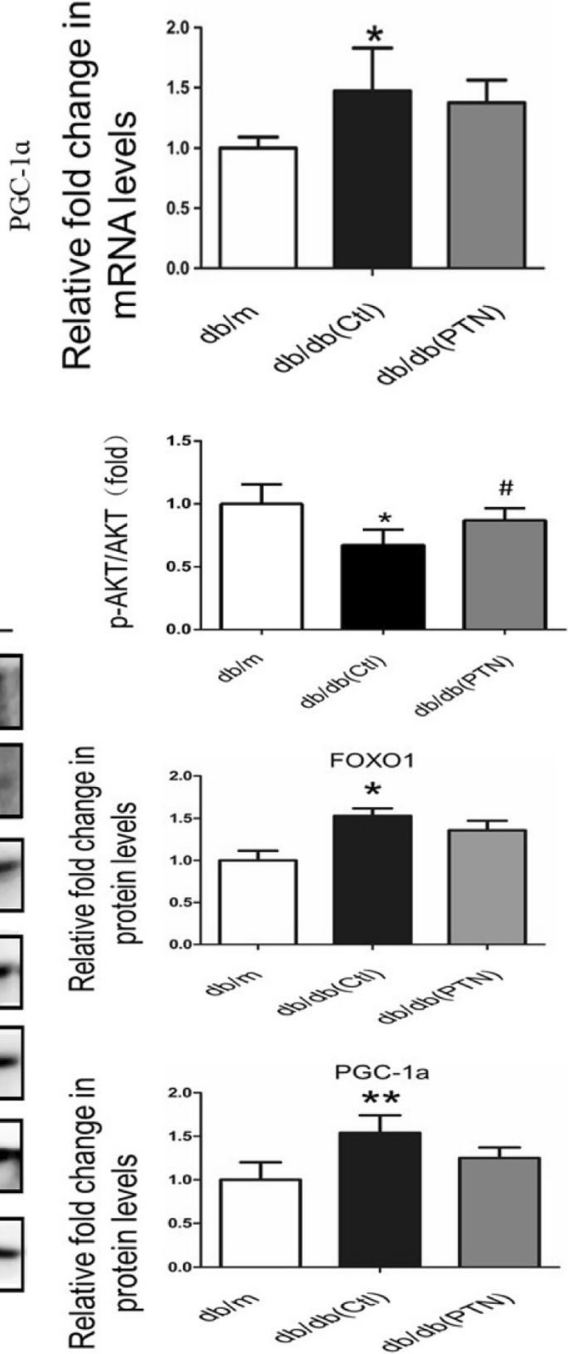

Fig. 5. Expression of insulin signaling and downstream gene in mice kidney. a Real-time PCR of FOXO1 and PGC-1a in renal cortex from db/m, db/db $(\mathrm{Ctl})$, and db/db (PTN) mice kidney. b Western blots of p-IRS1/IRS1, p-AKT/AKT, FOXO1, and PGC-1a in renal cortex of db/m, db/db (Ctl), and db/db (PTN) mice. $* P<0.05$ and $* * P<0.01$ compared with $\mathrm{db} / \mathrm{m}$. $\# P<0.05$ compared with $\mathrm{db} / \mathrm{db}(\mathrm{Ctl}) . n=6-8$ for each group.

Hence, this study aims to investigate whether renal inflammation-mediated insulin resistance contributes to the enhanced renal gluconeogenesis in T2DM. Unlike T2DM patients in vivo, few animal T2DM models have been used to study renal gluconeogenesis. Therefore, this study uses $\mathrm{db} / \mathrm{db}$ mice, which are considered to be the excellent T2DM model, and assumes that the renal gluconeogenesis in $\mathrm{db} / \mathrm{db}$ mice is intrinsically stimulated. Our findings suggest that the expression of inflammatory factors and gluconeogenic enzyme PEPCK is up-regulated in the renal cortex of both $\mathrm{db} /$ $\mathrm{db}$ mice and T2DM patients. In addition, the reduced insulin signaling while up-regulation of the downstream genes
FOXO1 and PGC-1a can be detected in the kidneys of db/ $\mathrm{db}$ mice compared with those in $\mathrm{db} / \mathrm{m}$ mice. Consistent with our hypothesis, NF- $\mathrm{KB}$ inhibitor PTN can mitigate renal inflammation and alleviate the renal insulin resistance. Moreover, it can also reduce the PEPCK expression, indicating that inflammation may potentially be one of the mechanisms responsible for the insulin resistance and the enhanced renal gluconeogenesis in T2DM.

The kidneys of T2DM patients can aggravate hyperglycemia through the enhanced gluconeogenesis and glucose reabsorption, both occurring in the proximal tubules [17]. However, the former is negatively modulated by insulin, 
while the latter is insulin independent [17]. Nonetheless, the kidney cannot release glucose through glycogenolysis [6], and it is also the main organ of gluconeogenesis in addition to the liver [18]. Rate-limiting gluconeogenic enzymes, including PEPCK, FBPase, and G6Pase, are mainly expressed in the renal PTs and liver [19]. However, these rate-limiting enzymes are not regulated in the same way. For instance, PEPCK and G6Pase are mainly modulated by transcriptional regulation, whereas FBPase has been reported to be under the allosteric regulation [20,21]. Here, we find that the regulation of PEPCK and FBPase expression in both mice and human kidneys are maintained in the same way as previously observed in rat liver, indicating that these enzymes are regulated in a consistent manner among different organs and species. Indeed, we have detected the enhanced renal expression of PEPCK, but not FBPase and G6Pase in both diabetics and $\mathrm{db} /$ $\mathrm{db}$ mice, suggesting that PEPCK may be the most susceptible gene. This is consistent with the report by Alejandro et al. on PTs from C57BL/6 mice fed with high-fat diet and in the kidneys from STZ-induced type 1 diabetic rats [22]. Typically, the lumen redistribution of PEPCK and FBPase is quite interesting in the PTs from diabetics and mice, indicating that the increased renal gluconeogenesis is mainly resulted from the enhanced luminal substrate uptake, which can be regulated by insulin signaling. PT hypertrophy and extracellular matrix (ECM) deposition are the early characteristics of diabetic nephropathy $(\mathrm{DN})$, which can be attributable to the hyperglycemia-induced inflammation and oxidative stress [23]. The capillaries peripheral to renal tubules (RTs) are important for the structure and function of RTs. However, renal tubulointerstitial lesion (TIL) and fibrosis will result in the loss of these capillaries during the development of DN [24]. Therefore, we speculate that the activated gluconeogenesis can move to the lumen side due to TIL, where abundant substrates are available.

Many factors would affect the renal gluconeogenesis, including hormones, substrates, and enzymes. The latest research from Kadowaki et al. suggested that renal gluconeogenesis could be cooperatively modulated through both the glucose reabsorption and insulin signaling in PTs [19]. Under fasting state, the restrained insulin signaling and reduced glucose reabsorption through sodium-glucose cotransporter 2 (SGLT2) on the luminal side can activate the renal gluconeogenesis [19]. Inversely, under postprandial state, expression of gluconeogenic genes is suppressed by both the increased glucose reabsorption and the activated insulin signaling [19]. Recently, growing attention has been paid to the mechanisms responsible for the enhanced renal gluconeogenesis in diabetes. For instance, Baverel et al. found that the increased circulating concentration of glucocorticoids and cAMP might play an important role in Zucker diabetic fatty rats, which was achieved through promoting the expression of key gluconeogenic genes [25]. However, Tiwari et al. had reported that the enhanced renal gluconeogenesis could be detected in PTs from C57BL/6 mice fed with high-fat diet and in the kidneys from STZ-induced type 1 diabetic rats through downregulating the expression of insulin receptor (IR) [18, 22]. Moreover, selective knockout of IR in the PTs of mice can promote hyperglycemia through the enhanced gluconeogenesis [26]. These studies reveal a crucial role of IR in PTs in affecting the systemic blood glucose levels; meanwhile, they have also indicated that the down-regulated expression of IR in PTs may further promote hyperglycemia through enhancing the renal gluconeogenesis. However, whether there is other reason remains unclear at present.

To our knowledge, insulin resistance can be resulted from cell modifications at both insulin receptor and postreceptor levels. Inflammatory stimulation may stimulate the phosphorylation of IRS-1 at serine-307, which can induce insulin resistance through activating the JNK and NF-kB pathways [13]. In fact, the relationship between inflammation and T2DM can be traced back to over one hundred years ago, when large doses (5.0-7.5 g/day) of sodium salicylate were first identified to reduce blood glucose levels in T2DM patients [13]. However, people were not well aware of the importance of insulin resistance in the pathogenesis of T2DM. Therefore, the improved insulin sensitivity had not been regarded as an underlying mechanism for reducing blood glucose at that time. Until 1993, scientists found that inflammation could directly induce insulin resistance, which promoted the restudy of the antihyperglycemic effects of salicylates. Moreover, it was demonstrated that the related mechanism was the activation inhibition of the NF$\mathrm{KB}$ signaling pathway $[27,28]$. It is generally known that T2DM is characterized by the chronic and subacute inflammation and insulin resistance $[14,15]$. In our work, we have detected enhanced activation of the NF- KB pathway and macrophage infiltration in $\mathrm{db} / \mathrm{db}$ mouse kidneys. To check whether inflammation contributes to the enhanced renal gluconeogenesis, we have used the NF-kB inhibitor parthenolide to reduce inflammation. Parthenolide, a sesquiterpene lactone that can be easily extracted from feverfew, has been identified to directly inhibit the NF-KB p65 subunit and the IKK complex [29]. Consistent with our hypothesis, PTN can mitigate renal inflammation in $\mathrm{db} / \mathrm{db}$ mice. Moreover, it can also alleviate renal insulin resistance and down-regulate the expression of gluconeogenic enzyme PEPCK, indicating that inflammation can be one of the mechanisms responsible for renal insulin resistance and the enhanced renal 
gluconeogenesis in $\mathrm{db} / \mathrm{db}$ mice. However, PTN cannot distinctly reduce the systemic insulin resistance and blood glucose levels, which may be attributed to the low dose of PTN. The diabetic $\mathrm{db} / \mathrm{db}$ mice are treated with a single intraperitoneal dose of $1 \mathrm{mg} / \mathrm{kg}$ PTN in the current study. This dose may be much lower than the therapeutic dose, since doctors will use large doses of salicylate (5.0-7.5 g/ day) to exert the antihyperglycemic effects in diabetics. However, such low dose of PTN has been able to dramatically reduce the renal inflammation and PEPCK expression.

Although renal gluoconeogenesis is known to be regulated by insulin signaling, the related molecular mechanism remains unclear until now. As is well known, expression of liver gluconeogenic genes is mainly modulated by insulin, especially by the suppression of forkhead transcription factor1. FOXO1 is a transcription factor that plays an important role in regulating gluconeogenesis. It can induce the expression of gluconeogenic genes through binding to the promoter regions of PEPCK and G6Pase [30,31]. The activation of insulin signaling conducted by IR/IRSs and AKT will stimulate the nuclear exclusion and degradation of FOXO1, consequently suppressing the expression of gluconeogenic genes [32, 33]. Kadowaki et al. had previously reported that specific knockout of IRS1/2 in mouse liver would promote the expression of hepatic gluconeogenic genes, which could be reversed by the inactivation of FOXO1 [34]. Another important gene is peroxisome proliferator-activated receptor gamma coactivator 1-alpha (PGC-1 $\alpha$ ), which works as a transcription coactivator in regulating the expression of gluconeogenic genes. Encouragingly, the latest results from Kadowaki et al. [19] reported that insulin would suppress the expression of gluconeogenic genes in HK-2 cells, together with the phosphorylation and inactivation of FOXO1. In addition, the inactivation of PGC- $1 \alpha$ by glucose deacetylation could also restrain the expression of gluconeogenic genes. These results demonstrate that the expression of renal gluconeogenic genes can be negatively regulated by both the inactivation of FOXO1 and PGC- $1 \alpha$ in PTs. Therefore, we have focused our attention on these two key genes, which are also involved in insulin resistance and dysregulated glucose metabolism in the liver. Consistent with that in the liver, the expression of FOXO1 and PGC- $1 \alpha$ is up-regulated in the kidneys of $\mathrm{db} / \mathrm{db}$ mice. However, PTN cannot dramatically down-regulate the expression of FOXO1 and PGC-1a in the

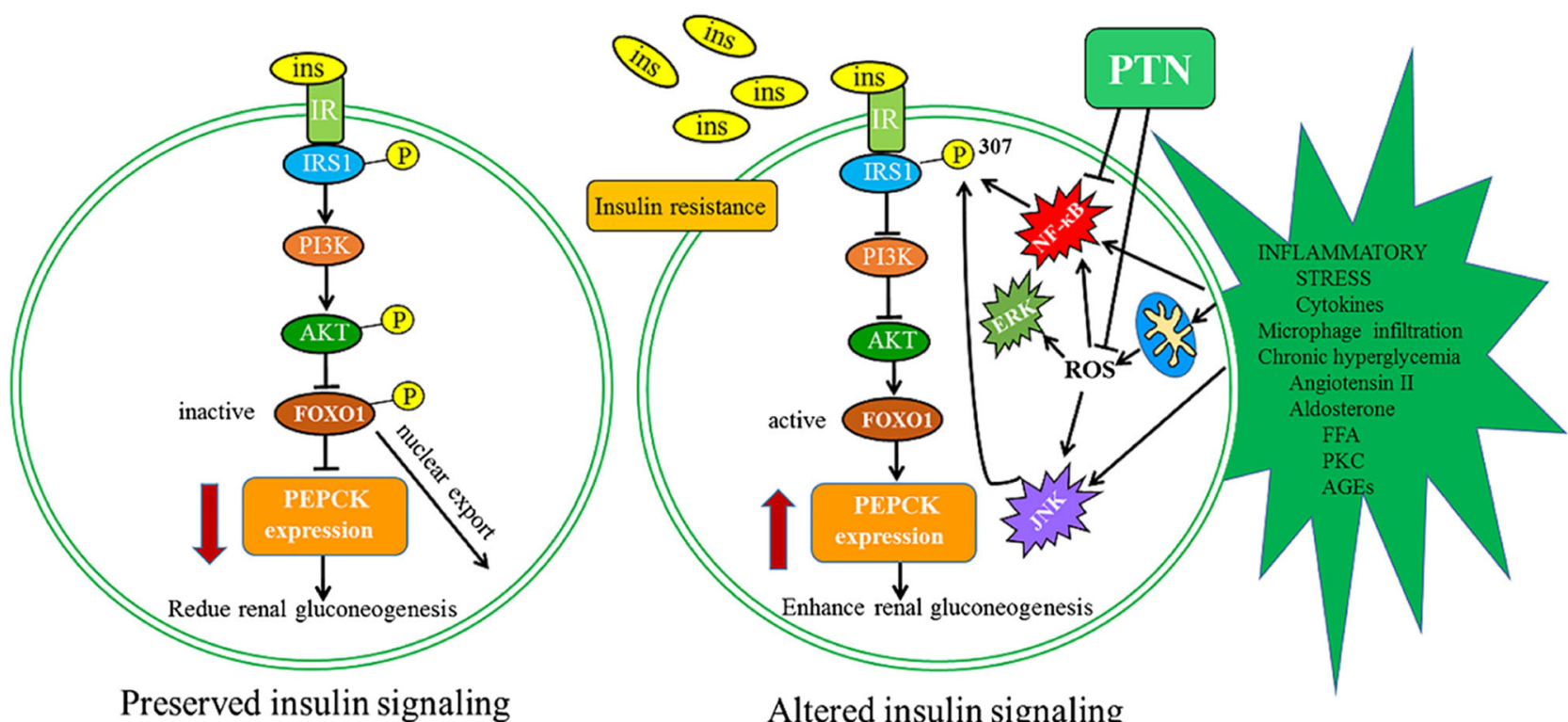

Normal PT

Diabetic PT

Scheme 1. Hypothetic model for altered insulin signaling and renal gluconeogenesis in renal proximal tubule (PT). In normal renal PT cells, insulin signaling conducted by IR/IRSs activates a series of intracellular signal transduction events which promotes the phosphorylation and activation of AKT. The activation of AKT promotes phosphorylation of FOXO1, leading to its translocation to the cytoplasm and degradation, consequently inhibiting gluconeogenic gene expression. PGC-1 $\alpha$ works as a transcription coactivator in the regulation of gluconeogenic gene expression. On the contrary, in diabetic PT cells, the local inflammatory stress may induce insulin resistance via ROS generation and activation of JNK and NF-kB pathways. In an insulin-resistance state, phosphatidylinositol 3 kinase (PI3K) and AKT are not activated, relieving FOXO1 phosphorylation and activating it, which in turn induce gluconeogenic gene expression. PTN could partially reverse this change by inhibiting NF-KB pathway. 
kidneys of $\mathrm{db} / \mathrm{db}$ mice. Actually, although FOXO-1 and PGC-1a are negatively regulated by $\mathrm{p}$-AKT, such regulation of FOXO-1 and PGC-1a activities is usually considered to be resulted from changes in phosphorylation level rather than those in its expression. There are many other molecules involved in the regulation of gluconeogenic gene expression in the liver, including sirtuin1 (Sirt1), FOXO3, and glycogen synthase kinase 3 beta (GSK3 $\beta$ ). Nevertheless, the roles of these genes in renal gluconeogenic gene expression should be further studied (Scheme 1).

Taken together, our study has demonstrated a novel mechanism by which the renal inflammation-mediated insulin resistance contributes to the enhanced renal gluconeogenesis in T2DM. To the best of our knowledge, this is the first report to reveal the associations among inflammation, insulin resistance, and the enhanced renal gluconeogenesis in $\mathrm{db} / \mathrm{db}$ mice. Obesity and T2DM have been recognized to be characterized by the chronic and subacute inflammation and insulin resistance. Meanwhile, discovering drugs that can secondarily alleviate inflammation is of great clinical significance, and many studies have suggested that it is possible to directly target the inflammatory process via pharmacological interventions, so as to prevent insulin resistance in T2DM or other metabolic conditions. These means may promote the clinical benefits to a huge number of patients suffering from these disorders. Howev$\mathrm{er}$, it remains to be further investigated regarding whether inflammatory inhibitor can reduce the systemic blood glucose level, as well as the specific molecular mechanisms involved in the renal gluconeogenesis.

Funding Information This study was supported by the Natural Science Foundation of Beijing, China (Grant No. 7122143).

\section{COMPLIANCE WITH ETHICAL STANDARDS}

Conflict of Interest. The authors declare that they have no conflicts of interest.

Ethical Approval. All procedures performed in studies involving human participants were in accordance with the ethical standards of the Institutional and/or National Research Committee and the 1964 Helsinki Declaration as well as its later amendments or comparable ethical standards. All applicable international, national, and/or institutional guidelines for the care and use of animals were followed.

Informed Consent. Informed consent was obtained from all individual participants included in the study.
Open Access This article is distributed under the terms of the Creative Commons Attribution 4.0 International License (http://creativecommons.org/licenses/by/4.0/), which permits unrestricted use, distribution, and reproduction in any medium, provided you give appropriate credit to the original author(s) and the source, provide a link to the Creative Commons license, and indicate if changes were made.

\section{REFERENCES}

1. Tahrani, A.A., C.J. Bailey, P.S. Del, and A.H. Barnett. 2011. Management of type 2 diabetes: new and future developments in treatment. Lancet 378 (9786): 182-197.

2. Landau, B.R., J. Wahren, V. Chandramouli, W.C. Schumann, K. Ekberg, and S.C. Kalhan. 1996. Contributions of gluconeogenesis to glucose production in the fasted state. Journal of Clinical Investigation 98 (2): 378-385.

3. Gerich, J.E. 2000. Physiology of glucose homeostasis. Diabetes Obesity \& Metabolism 2 (6): 345-350.

4. Consoli, A., F. Kennedy, J. Miles, and J. Gerich. 1987. Determination of Krebs cycle metabolic carbon exchange in vivo and its use to estimate the individual contributions of gluconeogenesis and glycogenolysis to overall glucose output in man. Journal of Clinical Investigation 80 (5): 1303-1310.

5. Meyer, C., J.M. Dostou, S.L. Welle, and J.E. Gerich. 2002. Role of human liver, kidney, and skeletal muscle in postprandial glucose homeostasis. American Journal of Physiology. Endocrinology and Metabolism 282 (2): E419-E427.

6. Stumvoll, M., C. Meyer, A. Mitrakou, V. Nadkarni, and J.E. Gerich. 1997. Renal glucose production and utilization: new aspects in humans. Diabetologia 40 (7): 749-757.

7. Meyer, C., M. Stumvoll, V. Nadkarni, J. Dostou, A. Mitrakou, and J. Gerich. 1998. Abnormal renal and hepatic glucose metabolism in type 2 diabetes mellitus. Journal of Clinical Investigation 102 (3): 619-624.

8. Meyer, C., H.J. Woerle, J.M. Dostou, S.L. Welle, and J.E. Gerich. 2004. Abnormal renal, hepatic, and muscle glucose metabolism following glucose ingestion in type 2 diabetes. American Journal of Physiology. Endocrinology and Metabolism 287 (6): E1049-E1056.

9. Cersosimo, E., R.L. Judd, and J.M. Miles. 1994. Insulin regulation of renal glucose metabolism in conscious dogs. Journal of Clinical Investigation 93 (6): 2584-2589.

10. Cersosimo, E., P. Garlick, and J. Ferretti. 1999. Insulin regulation of renal glucose metabolism in humans. The American Journal of Physiology 276 (1 Pt 1): E78-E84.

11. Kida, K., S. Nakajo, F. Kamiya, Y. Toyama, T. Nishio, and H. Nakagawa. 1978. Renal net glucose release in vivo and its contribution to blood glucose in rats. Journal of Clinical Investigation 62 (4): 721-726.

12. Meyer, C., J. Dostou, V. Nadkarni, and J. Gerich. 1998. Effects of physiological hyperinsulinemia on systemic, renal, and hepatic substrate metabolism. The American Journal of Physiology 275 (6 Pt 2): F915-F921.

13. Shoelson, S.E., J. Lee, and A.B. Goldfine. 2006. Inflammation and insulin resistance. Journal of Clinical Investigation 116 (7): 1793-1801.

14. Kanwar, Y.S., L. Sun, P. Xie, F.Y. Liu, and S. Chen. 2011. A glimpse of various pathogenetic mechanisms of diabetic nephropathy. Аnпиal Review of Pathology 6: 395-423. 
15. Qiu, Y.Y., and L.Q. Tang. 2016. Roles of the NLRP3 inflammasome in the pathogenesis of diabetic nephropathy. Pharmacological Research $114(251-264$.

16. Liu, Q., J. Jin, J. Ying, M. Sun, Y. Cui, L. Zhang, B. Xu, Y. Fan, and Q. Zhang. 2015. Frequent epigenetic suppression of tumor suppressor gene glutathione peroxidase 3 by promoter hypermethylation and its clinical implication in clear cell renal cell carcinoma. International Journal of Molecular Sciences 16 (5): 10636-10649.

17. DeFronzo, R.A., L. Norton, and M. Abdul-Ghani. 2017. Renal, metabolic and cardiovascular considerations of SGLT2 inhibition. Nature Reviews Nephrology 13 (1): 11-26.

18. Pandey, G., K. Shankar, E. Makhija, A. Gaikwad, C. Ecelbarger, A. Mandhani, A. Srivastava, and S. Tiwari. 2017. Reduced insulin receptor expression enhances proximal tubule gluconeogenesis. Journal of Cellular Biochemistry 118 (2): 276-285.

19. Sasaki, M., T. Sasako, N. Kubota, Y. Sakurai, I. Takamoto, T. Kubota, R. Inagi, G. Seki, M. Goto, K. Ueki, M. Nangaku, T. Jomori, and T. Kadowaki. 2017. Dual regulation of gluconeogenesis by insulin and glucose in the proximal tubules of the kidney. Diabetes 66 (9): 2339-2350.

20. Bertinat, R., J.P. Pontigo, M. Perez, I.I. Concha, M.R. San, J.J. Guinovart, J.C. Slebe, and A.J. Yanez. 2012. Nuclear accumulation of fructose 1,6-bisphosphatase is impaired in diabetic rat liver. Journal of Cellular Biochemistry 113 (3): 848-856.

21. Pilkis, S.J., M.R. El-Maghrabi, M. McGrane, J. Pilkis, and T.H. Claus. 1982. Regulation by glucagon of hepatic pyruvate kinase, 6-phosphofructo 1-kinase, and fructose-1,6-bisphosphatase. Federation Proceedings 41 (10): 2623-2628.

22. Gatica, R., R. Bertinat, P. Silva, D. Carpio, M.J. Ramirez, J.C. Slebe, M.R. San, F. Nualart, J.M. Campistol, C. Caelles, and A.J. Yanez. 2013. Altered expression and localization of insulin receptor in proximal tubule cells from human and rat diabetic kidney. Journal of Cellular Biochemistry 114 (3): 639-649.

23. Brezniceanu, M.L., F. Liu, C.C. Wei, I. Chenier, N. Godin, S.L. Zhang, J.G. Filep, J.R. Ingelfinger, and J.S. Chan. 2008. Attenuation of interstitial fibrosis and tubular apoptosis in $\mathrm{db} / \mathrm{db}$ transgenic mice overexpressing catalase in renal proximal tubular cells. Diabetes 57 (2): 451-459.

24. Singh, D.K., P. Winocour, and K. Farrington. 2008. Mechanisms of disease: the hypoxic tubular hypothesis of diabetic nephropathy. Nature Clinical Practice. Nephrology 4 (4): 216-226.

25. Eid, A., S. Bodin, B. Ferrier, H. Delage, M. Boghossian, M. Martin, G. Baverel, and A. Conjard. 2006. Intrinsic gluconeogenesis is enhanced in renal proximal tubules of Zucker diabetic fatty rats. Journal of the American Society of Nephrology 17 (2): 398-405.

26. Tiwari, S., R.S. Singh, L. Li, S. Tsukerman, M. Godbole, G. Pandey, and C.M. Ecelbarger. 2013. Deletion of the insulin receptor in the proximal tubule promotes hyperglycemia. Journal of the American Society of Nephrology 24 (8): 1209-1214.

27. Hundal, R.S., K.F. Petersen, A.B. Mayerson, P.S. Randhawa, S. Inzucchi, S.E. Shoelson, and G.I. Shulman. 2002. Mechanism by which high-dose aspirin improves glucose metabolism in type 2 diabetes. Journal of Clinical Investigation 109 (10): 1321-1326.

28. Shoelson, S.E., J. Lee, and M. Yuan. 2003. Inflammation and the IKK beta/I kappa B/NF-kappa B axis in obesity- and diet-induced insulin resistance. International Journal of Obesity and Related Metabolic Disorders 27 (Suppl 3): S49-S52.

29. Ghantous, A., H. Gali-Muhtasib, H. Vuorela, N.A. Saliba, and N. Darwiche. 2010. What made sesquiterpene lactones reach cancer clinical trials? Drug Discovery Today 15 (15-16): 668-678.

30. Nakae, J., W.R. Biggs, T. Kitamura, W.K. Cavenee, C.V. Wright, K.C. Arden, and D. Accili. 2002. Regulation of insulin action and pancreatic beta-cell function by mutated alleles of the gene encoding forkhead transcription factor Foxo1. Nature Genetics 32 (2): 245253.

31. Hall, R.K., T. Yamasaki, T. Kucera, M. Waltner-Law, R. O'Brien, and D.K. Granner. 2000. Regulation of phosphoenolpyruvate carboxykinase and insulin-like growth factor-binding protein-1 gene expression by insulin. The role of winged helix/forkhead proteins. Journal of Biological Chemistry 275 (39): 30169-30175.

32. Brunet, A., A. Bonni, M.J. Zigmond, M.Z. Lin, P. Juo, L.S. Hu, M.J. Anderson, K.C. Arden, J. Blenis, and M.E. Greenberg. 1999. Akt promotes cell survival by phosphorylating and inhibiting a Forkhead transcription factor. Cell 96 (6): 857-868.

33. Nakae, J., B.C. Park, and D. Accili. 1999. Insulin stimulates phosphorylation of the forkhead transcription factor FKHR on serine 253 through a Wortmannin-sensitive pathway. Journal of Biological Chemistry 274 (23): 15982-15985.

34. Kubota, N., T. Kubota, S. Itoh, H. Kumagai, H. Kozono, I. Takamoto, T. Mineyama, H. Ogata, K. Tokuyama, M. Ohsugi, T. Sasako, M. Moroi, K. Sugi, S. Kakuta, Y. Iwakura, T. Noda, S. Ohnishi, R. Nagai, K. Tobe, Y. Terauchi, K. Ueki, and T. Kadowaki. 2008. Dynamic functional relay between insulin receptor substrate 1 and 2 in hepatic insulin signaling during fasting and feeding. Cell $\mathrm{Me}$ tabolism 8 (1): 49-64. 